\title{
Bipolaronic phase in polar semiconductor quantum dots: An all-coupling approach
}

\author{
Phani Murali Krishna ${ }^{a}$, Soma Mukhopadhyay ${ }^{b, 1}$, Ashok Chatterjee ${ }^{b, *, 2}$ \\ ${ }^{a}$ School of Physics, University of Hyderabad, Hyderabad 500046, India \\ ${ }^{\mathrm{b}}$ Department of Physics, Bilkent University, Ankara, Turkey \\ Received 24 April 2004; accepted 1 September 2006 \\ Available online 22 September 2006 \\ Communicated by J. Flouquet
}

\begin{abstract}
An all-coupling variational calculation has been performed to explore the formation and stability of a bipolaron in a polar semiconductor quantum dot. It has been shown that quantum confinement in general leads to a broadening of the bipolaron stability region. It has been furthermore shown for the first time that stable bipolarons can exist in realistic parabolic quantum dots of polar semiconductors like GaAs, CdS, CdTe and CdSe if they are fabricated in certain range of sizes.
\end{abstract}

(C) 2006 Elsevier B.V. All rights reserved.

PACS: 71.38.-k; 63.20.Kr; 73.21.Hb

Keywords: Quantum dot; Polaronic effects; Bipolaron

Much effort has lately gone into exploring the polaronic effects in quantum dots (see [1] and references therein). It has been shown that electron-longitudinal-optical (LO) phonon interaction has pronounced effects on the electronic properties of quantum dots. Several investigations have also been made to study the formation and stability of bipolarons in a quantum dot. A bipolaron is a bound pair of two polarons with a common cloud of virtual phonons. Bipolarons are known to be important in semiconducting glasses in which diamagnetism is a rule rather than an exception [2]. The discovery of high temperature superconductivity in $\mathrm{CuO}_{2}$ based layered ceramic materials [3] and subsequent proposal of bipolaronic mechanism [4] for pairing has made the bipolaron problem most fascinating in recent years. In the case of quantum dots, the bipolaron problem was first investigated by Mukhopadhyay and Chatterjee [5] and it

\footnotetext{
* Corresponding author.

E-mail address: ashok@fen.bilkent.edu.tr (A. Chatterjee).

1 On leave from Department of Physics, Shadan Institute of P. G. Studies, Kharatabad, Hyderabad, India.

2 On leave from School of Physics, University of Hyderabad, Hyderabad 500 046, India.
}

was shown that in the strong coupling limit the confining potential of the quantum dot reduces the stability of the bipolaron. Essentially similar results were also observed in [6]. Pokatilov et al. [7] have investigated the stability of bipolarons in a spherical quantum dot with parabolic confinement by applying Feynman's variational principle and calculated the bipolaron binding energy, number of phonons in a bipolaron cloud and the bipolaron radius. They have shown that in a quantum dot bipolaron states are possible even for intermediate values of the electronphonon coupling constant, $\alpha(\alpha \sim 2)$. They have also shown that the binding energy passes through a maximum for a certain value of the confinement length. Recently, Senger and Ercelebi [8] have made a very interesting investigation on the stability of a bipolaron in spherical quantum dots using a single Hamiltonian and a variational method and have obtained a broader range of stability than shown in [5]. Because of the conflicting conclusions obtained by different groups, the bipolaron problem in a quantum dot has of late become a very interesting problem and has thrown up a new challenge to the theorists. It is therefore worthwhile to take up this problem and make a thorough investigation. The purpose of the present Letter is to make an attempt in this direction. Furthermore, to our knowledge, no 
investigation has so far been reported on the bipolaronic stability in realistic quantum dots. In the present Letter we shall show for the first time that stable bipolarons can indeed form in realistic quantum dots of polar semiconductors like GaAs, CdS, $\mathrm{CdSe}$ and CdTe. We shall also show that bipolarons cannot form in an InSb quantum dot.

In [5] the analysis was based on the assumption that in the unbound phase there exists no interaction between the two individual polarons which (though a reasonable approximation in bulk systems) is certainty not true in quantum dot structures. In the present Letter we therefore include a Coulomb correlation term in the two-polaron energy in the unbound phase while defining the bipolaron binding energy. Furthermore, our present calculation is based on the Lee-Low-Pines-Huybrechts (LLPH) method [9] and is therefore valid for the entire range of the coupling constant and arbitrary confinement. We shall show that inclusion of Coulomb correlation energy in the unbound phase brings in a dramatic and qualitative change in the results.

The Hamiltonian for a system of two conduction band electrons of mass $m$, each interacting through the Coulomb interaction in an $\mathrm{N}$-dimensional polar semiconductor quantum dot and also interacting with the dispersionless LO phonons of frequency $\omega_{0}$ of the system can be written in Feynman units $\left(\hbar=m=\omega_{0}=1\right)[10]$ as

$$
\begin{aligned}
\mathcal{H}= & -\frac{1}{2} \sum_{i=1}^{2} \nabla_{\vec{r}_{i}}^{2}+\frac{1}{2} \omega^{2} \sum_{i=1}^{2} r_{i}^{2}+\frac{\beta}{\left|\vec{r}_{1}-\vec{r}_{2}\right|}+\sum_{\vec{q}} b_{\vec{q}}^{\dagger} b_{\vec{q}} \\
& +\sum_{i=1}^{2} \sum_{\vec{q}}\left[\xi_{q} e^{-\vec{q} \cdot \vec{r}} b_{\vec{q}}^{\dagger}+\text { h.c. }\right]
\end{aligned}
$$

where energy has been scaled by $\hbar \omega_{0}$ and length by $r_{0}=$ $\left(\hbar / m \omega_{0}\right)^{1 / 2}, \vec{r}_{1}$ and $\vec{r}_{2}$ are the dimensionless position vectors of the two electrons, $\omega$ is the dimensionless frequency of the harmonic confining potential, $\beta=e^{2} / \epsilon_{\infty}=\sqrt{2} \alpha /(1-\eta)$ with $\eta=\epsilon_{\infty} / \epsilon_{0}, \epsilon_{\infty}$ and $\epsilon_{0}$ being respectively the high frequency and static dielectric constants, $b_{\vec{q}}^{\dagger}\left(b_{\vec{q}}\right)$ is the creation (annihilation) operator for an LO phonon of wave vector $\vec{q}$ and frequency $\omega_{0}$ and $\xi_{q}$ is the electron-phonon interaction coefficient given by [11]

$\left|\xi_{q}\right|^{2}=\frac{\Gamma\left(\frac{N-1}{2}\right) 2^{N-\frac{3}{2}} \pi^{\frac{N-1}{2}}}{V_{n} q^{N-1}} \alpha$,

where $V_{N}$ is the volume of the $N$-dimensional crystal and $\alpha$ is the dimensionless electron-phonon coupling constant. We seek a variational solution of Eq. (1) for a singlet bipolaron within the framework of the LLPH method and choose a trial wave function of the form

$$
\begin{aligned}
\left|\Psi_{\mathrm{BP}}\right\rangle= & \exp \left[-\frac{i a}{2} \sum_{i=1}^{n} \sum_{\vec{q}} \vec{q} \cdot \vec{r}_{i} b_{\vec{q}}^{\dagger} b_{\vec{q}}\right] \\
& \left.\left.\times \exp \left[\sum_{\vec{q}}\left(f_{\vec{q}} b_{\vec{q}}^{\dagger}-f_{\vec{q}}^{*} b_{\vec{q}}\right)\right]|0\rangle\left|\Phi\left(\vec{r}_{1}, \vec{r}_{2}\right)\right| \zeta\right\rangle\right\rangle,
\end{aligned}
$$

where $a$ and $f_{q}$ are variational parameters, $|0\rangle$ is the zerophonon state, $|\zeta\rangle$ is the antisymmetric spin function for the two electrons corresponding to the singlet pairing and $\left|\Phi\left(\vec{r}_{1}, \vec{r}_{2}\right)\right\rangle$ is a symmetric two-electron state which we choose as

$$
\begin{aligned}
\left|\Phi\left(\vec{r}_{1}, \vec{r}_{2}\right)\right\rangle= & \left(\frac{\lambda^{N}\left(\lambda^{2}-b\right)^{\frac{N}{2}+1}}{N \pi^{N}}\right)^{1 / 2}\left|\vec{r}-\vec{r}_{2}\right| \\
& \times e^{\frac{b}{4}\left|\vec{r}_{1}-\vec{r}_{2}\right|^{2}} e^{-\frac{\lambda^{2}}{2}\left(r_{1}^{2}+r_{2}^{2}\right)},
\end{aligned}
$$

where $\lambda$ and $b$ are variational parameters. The variational energy is given by

$$
E_{\mathrm{BP}}=\left\langle\Psi_{\mathrm{BP}}|\mathcal{H}| \Psi_{\mathrm{BP}}\right\rangle
$$

which on minimization with respect to $f_{q}^{*}$ yields

$$
\begin{aligned}
f_{q}= & -\frac{2 \xi_{\vec{q}}}{\left(1+\frac{a^{2} q^{2}}{4}\right)}\left[1-\frac{q^{2}}{4 N\left(\lambda^{2}-b\right)}\right] \\
& \times e^{-\frac{q^{2}}{8}\left\{\frac{(1-a)^{2}}{\lambda^{2}}+\frac{1}{\lambda^{2}-b}\right\}}
\end{aligned}
$$

where we have assumed $\sum_{\vec{q}} \vec{q}\left|f_{q}\right|^{2}=0$ for a symmetric dot. Defining $\lambda_{2}^{2}=\lambda^{2}-b$ and $t^{2}=\lambda^{2} / \lambda_{2}^{2}=\lambda^{2} /\left(\lambda^{2}-b\right)$ as two new variational parameters in place of $\lambda$ and $b$, we finally get the bipolaron energy

$$
\begin{aligned}
E_{\mathrm{BP}}^{\mathrm{ND}}= & \frac{N}{4} \lambda_{2}^{2} t^{2}+\frac{N}{4} \lambda_{2}^{2}-\frac{1}{2}\left(1-\frac{2}{N}\right) \lambda_{2}^{2} \\
& +\frac{\beta \sqrt{2} \lambda_{2} \Gamma\left(\frac{N+1}{2}\right)}{N \Gamma\left(\frac{N}{2}\right)}+\frac{\omega^{2}}{2}\left[\frac{N}{2 \lambda_{2}^{2} t^{2}}+\frac{\frac{N}{2}+1}{\lambda_{2}^{2}}\right] \\
& -\frac{4 \sqrt{2}}{\sqrt{\pi}} \frac{\Gamma\left(\frac{N-1}{2}\right)}{\Gamma\left(\frac{N}{2}\right)} \alpha \int_{0}^{\infty} d x \frac{\left(1-\frac{x^{2}}{N}\right)^{2} \lambda_{2} e^{-x^{2}\left\{\frac{(1-a)^{2}}{t^{2}}+1\right\}}}{\left(1+\alpha^{2} x^{2} \lambda_{2}^{2}\right)}
\end{aligned}
$$

which has to be minimized numerically with respect to $\lambda_{2}, a$ and $t$. We are interested here in the case $N=3$. When $a=0$ we get back the Landau-Pekar bipolaron ground state energy for a quantum dot [5] and when $\omega=0$ we have the bulk bipolaron energy results obtained by Chatterjee and Sil [12].

To determine the bipolaron binding energy we need to obtain the single polaron energy within the same approximation. The ground state polaron energy for a three-dimensional quantum dot with parabolic confinement calculated using the LLPH method is given by [13]

$$
E_{p}=\frac{3}{4} \mu^{2}+\frac{3}{4} \frac{\omega^{2}}{\mu^{2}}-\frac{1}{2 \sqrt{2} \pi^{2}} \alpha \int \frac{d \vec{q} e^{-\frac{\left(1-a^{\prime}\right)^{2}}{2 \mu^{2}} q^{2}}}{q^{2}\left(1+\frac{a^{\prime} q^{2}}{2}\right)},
$$

where $\mu$ and $a^{\prime}$ are the variational parameters to be obtained numerically.

The bipolaron binding energy for a quantum dot may be defined as

$$
B_{\mathrm{BP}}=\left(2 E_{p}+E_{c}\right)-E_{\mathrm{BP}}
$$

where $E_{c}$ is the Coulomb interaction energy between the individual polarons in the unbound phase. It is reasonable to assume that in the unbound phase the Coulomb correlation would keep the two polarons as much away from each other as possible to 


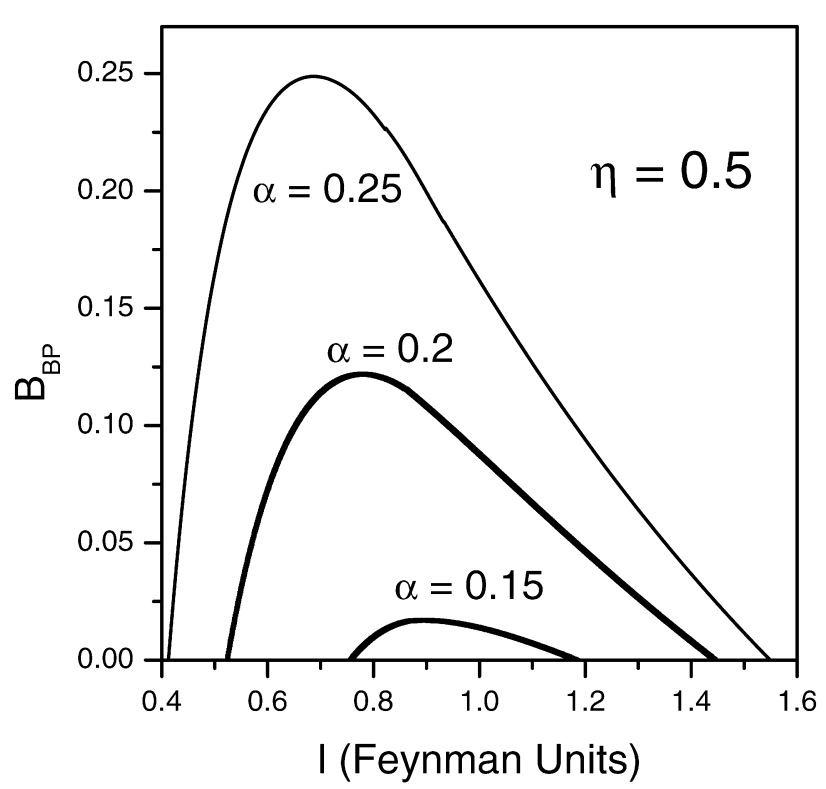

Fig. 1. $B_{\mathrm{BP}}$ (in Feynman units) as a function of the confinement length $l$ for different values of the electron-phonon coupling constant $\alpha$ and for $\eta=0.5$.

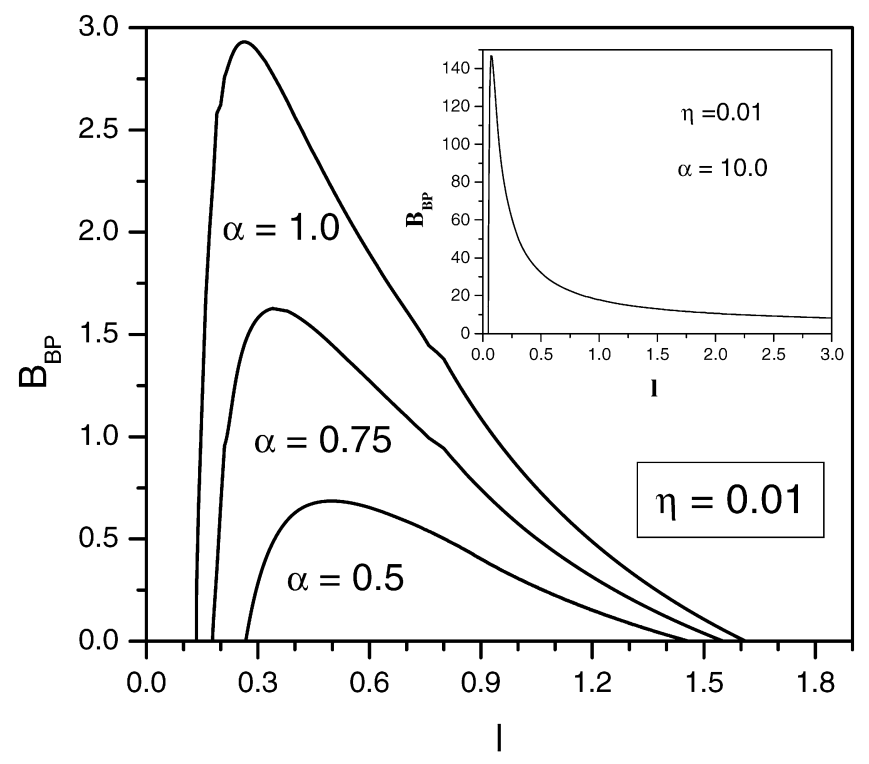

Fig. 2. $B_{\mathrm{BP}}$ (in Feynman units) as a function of the confinement length $l$ (in Feynman units) for different values of $\alpha$ and for $\eta=0.01$. The inset shows the variation of $B_{\mathrm{BP}}$ for $\alpha=10.0$.

lower the energy. So we assume $E_{c}=\beta / l$. The bipolaronic stability criteria are now obtained by demanding $B_{\mathrm{BP}}>0$. The binding energy of an all-coupling bipolaron is found to depend on three parameters $\eta, l$ and $\alpha$.

In Fig. 1 we show the variation of the bipolaron binding energy $B_{\mathrm{BP}}$ as a function of $l$ for several values of $\alpha$ and for $\eta=0.5$. In Fig. 2 we show the behaviour for the case of $\eta=0.01$. Comparison with the results of [5] shows that inclusion of Coulomb correlation between the individual polarons in the unbound phase leads to an enormous increase in the bipolaron binding energy at smaller values of confinement length l. As a result, it is clear that even for smaller values of $\alpha$, there exist regions in which bipolaronic state may be possible.

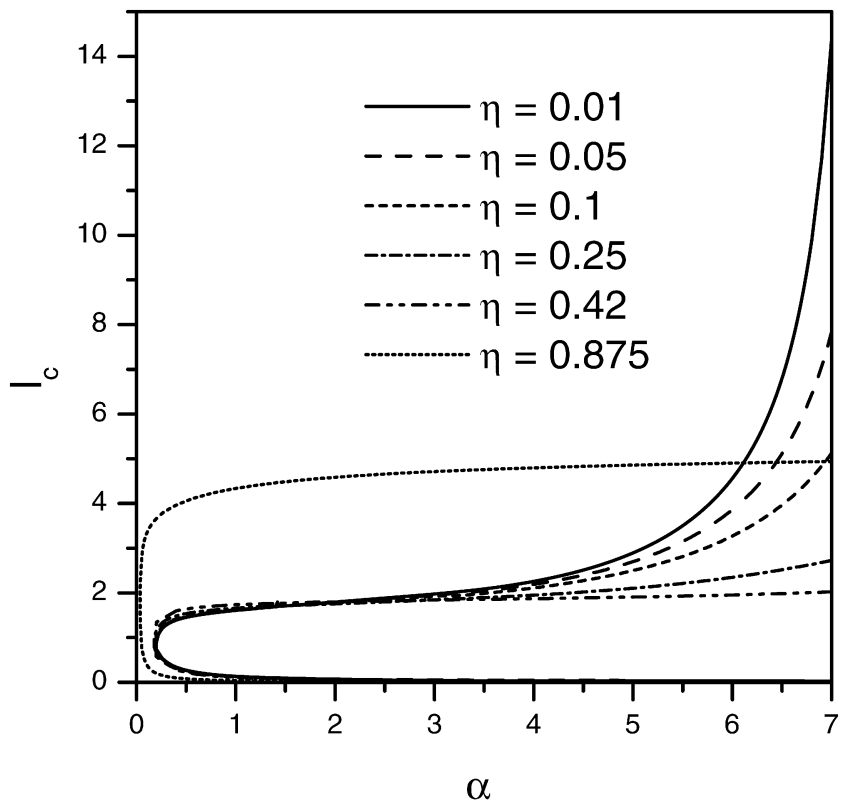

Fig. 3. $l_{c}$ (in Feynman units) as a function of the electron-phonon coupling constant $\alpha$ for different values of $\eta$.

It is well known that for weak electron-phonon coupling, stable bipolarons cannot form in bulk systems. However, we find here that as the confinement length is reduced below a certain value (depending on the coupling constant) the bipolaron binding energy starts increasing, attains a maximum and then decreases very fast and eventually below a certain confinement length a bipolaron breaks up into two individual polarons. Similar behaviour was also observed by Pokatilov et al. [7]. Thus we can define two critical confinement lengths in general, $l_{c_{1}}$ and $l_{c_{2}}$ for each value of $\alpha . l_{c_{1}}$ may be called the lower critical confinement length and $l_{c_{2}}$ the upper critical confinement length. $B_{\mathrm{BP}}$ is positive if the confinement length $l$ is between $l_{c_{1}}$ and $l_{c_{2}}$. The inset in Fig. 2 indicates that for $\alpha=10$, as $l$ increases and becomes large, the binding energy asymptotically saturates to a constant value which is essentially the strong-coupling bulk limit. Thus, when $\alpha$ is very large, $l_{c_{2}}$ is of the order of the size of the system. As $\alpha$ decreases, $l_{c_{2}}$ also decreases, but $l_{c_{1}}$ increases (although not so rapidly) and consequently the bipolaronic phase shrinks and below a certain critical value of $\alpha\left(\alpha_{c}\right)$ the bipolaronic phase vanishes and therefore below this value of $\alpha$, stable bipolarons cannot exist. For example, we find that for $\eta=0.875, \alpha_{c}=0.037$. This has been shown in Fig. 3 from which we can also find, given the value of $\eta$ and $\alpha$, the range of the confinement length within which one can expect to observe stable bipolaronic states in a quantum dot. Below a certain critical confinement length one cannot have the formation of bipolarons. One can qualitatively understand this behaviour in quite a simple way. When electron-electron separation is of the order of the polaron size or larger, then the bipolaron formation depends on whether the phonon-mediated attractive interaction overcomes the Coulomb repulsion or not. However when the size of the quantum dot is smaller than a certain critical value (which is close to zero), the electron-electron separation will also be very small, and in this limit the direct Coulomb repul- 


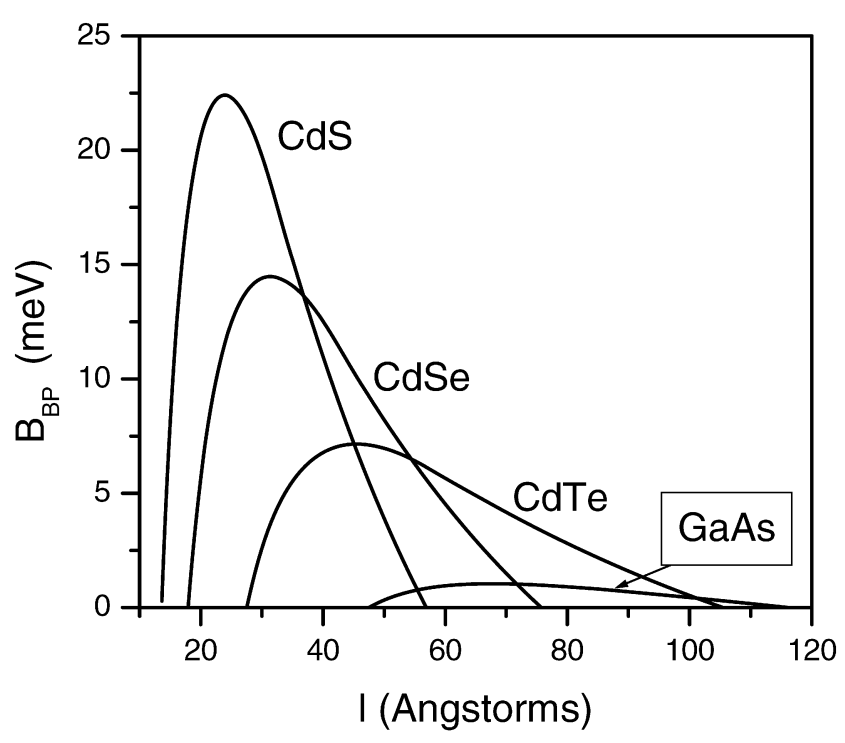

Fig. 4. $B_{\mathrm{BP}}$ (in meV) as a function of the confinement length $l(\AA)$ for a few real polar semiconductor quantum dots.

sion becomes infinitely large, although the phonon-mediated attractive interaction still remains finite and thus bipolaron binding does not take place howsoever large the value of $\alpha$ may be. In this limit a bipolaron breaks up into individual polarons.

We have finally applied our theory to realistic quantum dots of polar semiconductors like GaAs, CdTe, CdSe, CdS and InSb. We have shown the bipolaron binding energies in these quantum dots in Fig. 4. It is clearly evident that bipolarons can exist in several semiconductor quantum dots if we prepare them in appropriate sizes. In $\mathrm{CdS}$ the bipolaron seems to be most stable amongst the materials we studied. In GaAs the bipolaron is barely stable while we also find that bipolarons cannot exist in InSb quantum dot. We believe it is a very interesting theoretical observation and should be tested experimentally. One of the possible ways to verify this theoretical prediction would be to perform a magneto-optical experiment and determine the cyclotron mass which will tell us unequivocally the nature of the quasiparticles in the system, i.e., whether they are individual polarons or bound bipolarons. One can also perform tunneling experiments in a quantum dot array wherefrom the charge of the tunneling particles will shed light on the nature of the quasiparticles. There could also be a few other experiments as well to test the veracity of the present theoretical prediction. The important point is that if the present prediction comes true, it can have many useful implications, one of the most important among them being the possibility of superconductivity in quantum dots induced by Bose-Einstein condensation of bipolarons.

\section{Acknowledgements}

One of the authors (R.P.M.K.) would like to gratefully acknowledge the financial support from the University of Hyderabad under the UPE (University with Potential for Excellence) programme of UGC and also from the CSIR, India.

\section{References}

[1] A. Chatterjee, S. Mukhopadhyay, Acta Phys. Pol. B 32 (2001) 473.

[2] P.W. Anderson, Phys. Rev. Lett. 34 (1975) 953.

[3] J.G. Bednorz, K.A. Müller, Z. Phys. 64 (1986) 189.

[4] A.S. Alexandrov, N.F. Mott, Rep. Prog. Phys. 57 (1994) 1197; A. Chatterjee, S. Sil, Mod. Phys. Lett. B 6 (1992) 959.

[5] S. Mukhopadhyay, A. Chatterjee, J. Phys.: Condens. Matter 8 (1996) 4017.

[6] R.T. Senger, A. Ercelebi, Eur. Phys. J. B 16 (2000) 439.

[7] E.P. Pokatilov, V.M. Fomin, J.T. Devreese, S.N. Balaban, S.N. Klimin, J. Phys.: Condens. Matter 11 (1999) 9033.

[8] R.T. Senger, A. Ercelebi, J. Phys.: Condens. Matter 14 (2002) 5549.

[9] W.J. Huybrechts, J. Phys. C 10 (1977) 3761; A. Chatterjee, Ann. Phys. (N.Y.) 202 (1990) 297.

[10] R. Feynman, Phys. Rev. 97 (1995) 660.

[11] F.M. Peeters, X. Wu, J.T. Devreese, Phys. Rev. B 33 (1986) 3926.

[12] A. Chatterjee, S. Sil, Int. J. Mod. Phys. B 7 (1993) 4763.

[13] S. Mukhopadhyay, A. Chatterjee, J. Phys.: Condens. Matter 11 (1999) 2071. 